\title{
Cardiomiopatia hipertrófica, uma importante causa de morte súbita em jovens:
}

\section{revisão integrativa}

\author{
Hypertrophic cardiomyopathy, an important cause of sudden death in young: integrative review \\ Miocardiopatía hipertrófica, una causa importante de muerte súbita en jóvenes: revisión
}

integradora

Thiago do Nascimento Sousa

ORCID: https://orcid.org/0000-0001-6515-3321 Instituto Tocantinense Presidente Antônio Carlos, Brasil

E-mail: sousathiago456@gmail.com

Higor César Parrião Lustosa

ORCID: https://orcid.org/0000-0002-9754-8472 Instituto Tocantinense Presidente Antônio Carlos, Brasil E-mail: higor.cesar7@gmail.com

Hian Costa Távora

ORCID: https://orcid.org/0000-0002-0204-6702 Instituto Tocantinense Presidente Antônio Carlos, Brasil hianctavora@gmail.com

Marcos André de Sousa Godinho ORCID: https://orcid.org/0000-0002-8456-7439 Instituto Tocantinense Presidente Antônio Carlos, Brasil E-mail: marcosangodinho@outlook.com

Marcello Augustus de Sena

ORCID: https://orcid.org/0000-0001-9652-5538 Instituto Tocantinense Presidente Antônio Carlos, Brasil

E-mail: marcellosena1@gmail.com

\begin{abstract}
Resumo
A Cardiomiopatia Hipertrófica $(\mathrm{CMH})$ é a doença cardíaca de base genética e transmissão hereditária mais comum, tendo sido identificados inúmeras mutações nos genes do sarcômero cardíaco. A principal característica da doença é a hipertrofia ventricular esquerda (HVE) de morfologias diversas, sem outras causas que expliquem a condição. Suas manifestações clínicas são variadas de acordo com as mutações de cada indivíduo e/ou família; o paciente pode apresentar-se na forma assintomática, ou evoluir com desfechos como arritmias, estenose aórtica subvalvar, insuficiência cardíaca (IC) e, até mesmo, morte súbita. Assim, o objetivo deste trabalho é abordar as principais manifestações clínicas da CMH. Foi realizada uma Revisão Integrativa da Literatura, com busca nas bases de dados Scielo, LILACS, UpToDate e MEDLINE. Foram incluídos os artigos publicados de 2015 a 2021, na íntegra e disponíveis eletronicamente em Inglês, espanhol ou Português. Foram excluídos os artigos que se repetiam, disponibilizados apenas em resumo ou sem relação com o objetivo do estudo. Encontrou-se 17796 publicações somando todas as plataformas, após empregar os critérios de inclusão e exclusão e utilizar os descritores restaram 3187 artigos; em seguida, a partir da leitura dos títulos e/ou resumos foram selecionados e analisados 25 artigos. Portanto, entende-se que a $\mathrm{CMH}$ é uma patologia de manifestação silenciosa em muitos casos, tornando a triagem essencial para detectar a doença precocemente e permitir intervenção médica antecipada, melhorando o prognóstico e a qualidade de vida dos pacientes; além disso, tem-se uma grande dificuldade de determinar o risco de desenvolver as manifestações clínicas, como a morte súbita cardíaca.
\end{abstract}

Palavras-chave: Cardiomiopatia hipertrófica; Morte súbita; Manifestações clínicas.

\begin{abstract}
The Hypertrophic Cardiomyopathy (HCM) is a cardiac disease from genetic bases and most common hereditary transmission, has been identified numerous mutations in the genes of the cardiac sarcomere. The major characteristic of the disease is left ventricular hypertrophy ( $\mathrm{LVH})$ of diferente morphologies, without other causes that explain the condition. Its clinical manifestations vary according to the mutations of each individual and/or family; the patient may present in the asymptomatic form, or evolve with outcomes such as arrhythmias, subvalvular aortic stenosis, heart failure and even sudden death. Thus, the objective of this work is to approach the main clinical manifestations of HCM. An Integrative Literature Review was carried out, searching the Scielo, LILACS, UpToDate and MEDLINE databases.
\end{abstract}


Articles published from 2015 to 2021, in full and available electronically in English, Spanish or Portuguese, were included. Articles that were repeated themselves, made available only in summary or unrelated to the purpose of the study, were excluded. Has been found 17.796 publications summing all platforms, after using the inclusion and exclusion criteria and using the descriptors 3187 articles remained; then, after reading the titles and/or abstracts, 25 articles were selected and analyzed. Therefore, it was understood that HCM is a silent disease in many cases, making screening essential to detect the disease early and allow precocious medical intervention, improving the prognosis and quality of life of patients; in addition, there is great difficulty in determining the risk of developing clinical manifestations, such as sudden cardiac death (SCD).

Keywords: Hypertrophic cardiomyopathy; Sudden death; Clinical manifestations.

\section{Resumen}

La miocardiopatía hipertrófica $(\mathrm{MCH})$ es la enfermedad cardíaca de base genética y la transmisión hereditaria más común, y se han identificado numerosas mutaciones en los genes del sarcómero cardíaco. La principal característica de la enfermedad es la hipertrofia ventricular izquierda (HVI) de diferentes morfologías, sin otras causas que expliquen la condición. Sus manifestaciones clínicas varían según las mutaciones de cada individuo y / o familia; el paciente puede presentarse de forma asintomática o evolucionar con resultados como arritmias, estenosis aórtica subvalvular, insuficiencia cardíaca e incluso muerte súbita. Así, el objetivo de este trabajo es abordar las principales manifestaciones clínicas de la MCH. Se realizó una Revisión Integrativa de Literatura, buscando en las bases de datos Scielo, LILACS, UpToDate y MEDLINE. Se incluyeron artículos publicados de 2015 a 2021, en su totalidad y disponibles electrónicamente en inglés, español o portugués. Se excluyeron los artículos que se repitieron, que se pusieron a disposición solo en forma resumida o que no estaban relacionados con el propósito del estudio. Encontramos 17796 publicaciones sumando todas las plataformas, luego de usar los criterios de inclusión y exclusión y usar los descriptores, quedaron 3187 artículos; luego, luego de la lectura de los títulos y / o resúmenes, se seleccionaron y analizaron 25 artículos. Por tanto, se entiende que la $\mathrm{MCH}$ es una enfermedad silenciosa en muchos casos, por lo que el cribado es fundamental para detectar precozmente la enfermedad y permitir una intervención médica precoz, mejorando el pronóstico y la calidad de vida de los pacientes; Además, existe una gran dificultad para determinar el riesgo de desarrollar manifestaciones clínicas, como la muerte súbita cardíaca (MSC).

Palabras clave: Miocardiopatía hipertrófica; Muerte súbita; Manifestaciones clínicas.

\section{Introdução}

A cardiomiopatia hipertrófica $(\mathrm{CMH})$ é a mais comum das doenças cardiovasculares de base eminentemente genética, mais de 900 mutações já foram descritas e, com frequência, são peculiares às famílias individuais. É transmitida por um traço mendeliano com padrão de herança autossômica dominante, levando a um risco de $50 \%$ para cada filho (a) da prole de indivíduos afetados herdarem a mutação e desenvolverem a doença, apresentando como causa diversos tipos de mutações em genes que codificam os constituintes proteicos do sarcômero cardíaco (Braunwald, 2013).

Vários estudos epidemiológicos têm descrito prevalência similar do fenótipo da CMH na população geral de 1:500, mas estudos ainda em andamento apontam que esse número pode ser reduzido para 1:200.

A CMH evidencia características morfológicas, funcionais e clínicas extremamente heterogêneas (Mattos, 2001), apresentando um curso clínico também bastante heterogêneo, evidenciando pacientes que permanecem de maneira sistemática na forma assintomática, enquanto outros apresentam morte súbita ou evoluem à insuficiência cardíaca. Trata-se de uma condição comum ao envolver morte súbita entre jovens e atletas profissionais, sendo responsável pelo desenvolvimento de insuficiência cardíaca e incapacidade física que se manifesta em qualquer idade, mas com ligeira prevalência na primeira década de vida.

Conforme abordado por Kumar (2020), destaca-se na patologia em questão o coração mostra-se pesado, com paredes espessadas e hipercontrátil, o que causa uma disfunção diastólica, levando a um enchimento diastólico anormal, e em cerca de um terço dos casos ocorre obstrução intermitente da via de saída do ventrículo. "O padrão clássico envolve o espessamento desproporcional do septo ventricular em relação à parede livre do ventrículo esquerdo (com uma proporção septo/parede livre maior do que 3:1), denominado hipertrofia septal assimétrica". 
Assim sendo, o tema mostra-se relevante devido a sua prevalência significativa, já que existe à necessidade de identificação precoce dos grupos em risco; adolescentes, adultos jovens e atletas. Este trabalho tem como objetivo abordar as principais manifestações clínicas da cardiomiopatia hipertrófica (CMH).

\section{Metodologia}

Ao alcance do objetivo proposto neste trabalho foi selecionado como método de escolha a Revisão Integrativa da Literatura, que determina o saber atualizado sobre um tema selecionado, se propondo a reconhecer, refletir e sintetizar resultados de estudos independentes sobre determinada temática (Duarte et al., 2014).

Trata-se de uma revisão integrativa da literatura do tipo qualitativa (Ludke \& Andre, 2013), com o objetivo de reunir e sintetizar resultados de estudos sobre manifestações clínicas da cardiomiopatia hipertrófica, de maneira ordenada e sistematizada, seguindo como pilar: identificação do tema e seleção da hipótese ou questão de pesquisa, estabelecimento de critérios para inclusão e exclusão de estudos, definição das informações a serem extraídas dos estudos selecionados, avaliação dos estudos incluídos na revisão integrativa, interpretação dos resultados, apresentação da revisão/síntese do conhecimento.

As bases de dados utilizadas para a pesquisa foram Scientific eletronic Library Online (Scielo), Literatura Latino Americana e o do Caribe em Ciências da Saúde (LILACS), UpToDate e Medical Literature Analysis and Retrievel System Online (MEDLINE) conforme descrito no Tabela 1. As plataformas foram selecionadas tendo como base o fato de possuírem os periódicos de maior relevância para a área da saúde.

Dentro das plataformas supracitadas, os Descritores Controlados de Ciências da Saúde (DeCS) utilizados foram "cardiomiopatia hipertrófica"; "morte súbita" e "manifestações clínicas" associadas aos operadores booleanos. Para o refinamento dos artigos foram estabelecidos os seguintes critérios de inclusão: artigos completos publicados na íntegra e disponíveis eletronicamente nos idiomas Inglês, Espanhol ou Português, houve recorte temporal considerando o período de 2015 a 2021 para a seleção dos artigos, possibilitando uma análise da evolução das pesquisas na temática. Excluíram-se as publicações que se repetiam nas bases de dados, publicados em ano inferior a 2015, disponibilizadas apenas em resumo ou que não tivessem relação com o objetivo da pesquisa e estivessem em outros idiomas.

Tabela 1. Estratégia de busca utilizada. Palmas (TO) Brasil, 2021.

\begin{tabular}{|c|c|c|c|c|}
\hline Acrônimo & LILACS & Scielo & UpTodate & MEDLINE \\
\hline \multirow{5}{*}{$\mathrm{P}$ (problema) } & "hypertrophic & "hypertrophic & "hypertrophic & "hypertrophic \\
\hline & cardiomyopathy" (mesh & cardiomyopathy" (mesh & cardiomyopathy" (mesh & cardiomyopathy" (mesh \\
\hline & terms) or "hypertrophic & terms) or "hypertrophic & terms) or "hypertrophic & terms) or "hypertrophic \\
\hline & cardiomyopathy" (text & cardiomyopathy" (text & cardiomyopathy" (text & cardiomyopathy" (text \\
\hline & word) & word) & word) & word) \\
\hline \multirow{4}{*}{ I (fenômeno de interesse) } & "clinical manifestations" & "clinical manifestations" & "clinical manifestations" & "clinical manifestations" \\
\hline & (mesh terms) or "clinical & (mesh terms) or "clinical & (mesh terms) or "clinical & (mesh terms) or "clinical \\
\hline & manifestations" (text & manifestations" (text & manifestations" (text & manifestations" (text \\
\hline & word) & word) & word) & word) \\
\hline \multirow{3}{*}{ Co (contexto) } & "sudden death" (mesh & "sudden death" (mesh & "sudden death" (mesh & "sudden death" (mesh \\
\hline & terms) or "athletes sudden & terms) or "athletes sudden & terms) or "athletes sudden & terms) or "athletes sudden \\
\hline & death" (text word) & death" (text word) & death" (text word) & death" (text word) \\
\hline
\end{tabular}

Fonte: Elaboração própria. 
Research, Society and Development, v. 10, n. 13, e336101321498, 2021

(CC BY 4.0) | ISSN 2525-3409 | DOI: http://dx.doi.org/10.33448/rsd-v10i13.21498

\section{Resultados}

Com base nos Descritores Controlados de Ciências da Saúde (DeCS) para as plataformas MEDLINE e LILACS, e pesquisa direta nas plataformas SciELO e UPTODATE, encontrou-se 17796 estudos, que correspondem a soma de todas as pesquisas realizadas, evidenciado na Tabela 2.

Tabela 2 - Resultados dos descritores.

\begin{tabular}{ccccc}
\hline DeCS & SciELO & MEDLINE & LILACS & UPtoDate \\
\hline CMH & 101 & 14941 & 497 & 151 \\
CMH + Morte súbita & 20 & 664 & 110 & 147 \\
CHM + Manifestações clínicas* & 27 & 980 & 9 & 149 \\
\hline
\end{tabular}

*considerados: insuficiência cardíaca, arritmias, estenose aórtica subvalvar... Fonte: Elaboração própria.

No intuito de desenvolver a pesquisa, foi empregada a utilização dos DeCS, pesquisa direta e critérios de inclusão e exclusão, encontrando-se 3187 artigos. Posteriormente, a partir da leitura dos títulos e/ou resumos, foram descartados os artigos que se repetiam nas plataformas de pesquisa e que destoavam do tema proposto, obtendo-se 25 artigos. As pesquisas levantadas e analisadas que apresentavam dados relevantes à revisão foram avaliadas por completo e selecionados para coleta de dados, conforme Tabela 3. Todos os artigos selecionados são apresentados na Tabela 4, assim como suas sínteses (Tabela $5)$.

Tabela 3: Exclusão e inclusão de artigos.

\begin{tabular}{lcccc}
\hline \multicolumn{1}{c}{ PLATAFORMAS } & ENCONTRADOS & SELECIONADOS & EXCLUÍDOS & ANALISADOS \\
\hline MEDLINE & 14941 & 2980 & 2973 & 7 \\
LILACS & 497 & 86 & 79 & 6 \\
UPTODATE & 447 & 73 & 67 & 6 \\
SCIELO & 148 & 48 & 42 & 6 \\
\hline
\end{tabular}

Fonte: Elaboração própria.

Tabela 4: Apresentação dos artigos incluídos na Revisão Integrativa.

\begin{tabular}{llr}
\hline $\mathbf{N}^{\circ}$ & \multicolumn{1}{c}{ TÍTULO } & AUTOR \\
\hline $\begin{array}{l}\text { Paro cardiaco repentino atribuído a miocardiopatía hipertrófica por uso de esteroides } \\
\text { anabólicos. Reporte de caso }\end{array}$ & Conde, M. D. et al. \\
2 & Cardiomiopatia Hipertrófica, Todos os Fenótipos em um & Arias, A. M. \\
3 & Morte Súbita na Cardiomiopatia Hipertrófica & Bittencourt, M. I. \\
4 & New Developments in Hypertrophic Cardiomyopathy & Cooper, R. M. et al.
\end{tabular}


6 Síncope na cardiomiopatia hipertrófica obstrutiva

Miectomía septal ampliada en la miocardiopatía hipertrófica obstructiva, resultados clínicos y evolución ecocardiográfica a mediano plazo

8 The Portuguese Registry of Hypertrophic Cardiomyopathy: Overall results

9

Identifying unmet clinical need in hypertrophic cardiomyopathy using national electronic health records

10 Miocardiopatia hipertrófica - reporte de caso

Avaliação da Função Ventricular Esquerda na Associação de Cardiomiopatia Hipertrófica e

Hipertensão Arterial Sistêmica pela Técnica de Strain

12

Caso 3/2019 - Homem Jovem com Quadro de Dispneia Intensa, Infiltrado Pulmonar, Área

Cardíaca Normal e Obliteração da Ponta do Ventrículo Esquerdo

13 Hypertrophic cardiomyopathy: Medical therapy for heart failure

14 Cardiomiopatia Hipertrófica - Revisão

15 Miocardiopatía hipertrófica: encareclínico y opciones de tratamiento

16 Hypertrophic cardiomyopathy: Clinical manifestations, diagnosis, and evaluation

17 Hypertrophic cardiomyopathy: Risk stratification for sudden cardiac death

18 Hypertrophic cardiomyopathy in children: Clinical manifestations and diagnosis

19

Hypertrophic cardiomyopathy: Management of ventricular arrhythmias and sudden cardiac death risk

20

Hypertrophic cardiomyopathy: Morphologic variants and the pathophysiology of left ventricular outflow tract obstruction

21

Discordância entre Diretrizes Internacionais sobre Critérios de Prevenção Primária de Morte Súbita na Cardiomiopatia Hipertrófica

22 Cardiomyopathies: An Overview

23 Gestion et résultats de la cardiomyopathie hypertrophique chez les jeunes adultes

24 Clinical characteristics and outcomes in childhood-onset hypertrophic cardiomyopathy

25 Discordant clinical features of identical hypertrophic cardiomyopathy twins
Licarião, E. G. D.

Vrancic, J. M.

Cardim, N. et al.

Pujades-Rodriguez, M. et al.

Loss, F. S.

Thereza Gil

Victor Sarli

Martin S. Maron

2019

Zanati S. G.

Caorsi W. R. et al

Martin S. Maron

Martin S. Maron

John L Jefferies, Thomas D.

Ryan, Martin S Maron

Martin S. Maron

Martin S. Maron

Mattos B. P.

Ciarambino T. et al.

Émilie Baron et al

Marston N. A. et al.

Giuliana G. Repetti et al.

Fonte: Elaboração própria. 
Research, Society and Development, v. 10, n. 13, e336101321498, 2021

(CC BY 4.0) | ISSN 2525-3409 | DOI: http://dx.doi.org/10.33448/rsd-v10i13.21498

Tabela 5: Apresentação da síntese dos artigos incluídos.

\begin{tabular}{ll}
\hline $\mathbf{N}^{\circ}$ & \multicolumn{1}{c}{ AMOSTRA/OBJETIVO } \\
\hline & $\begin{array}{l}\text { Relatar um caso clínico incomum em nosso ambiente } \\
\text { hospitalar de um paciente com uso crônico de } \\
\text { esteróides anabólicos androgênicos e cardiomiopatia } \\
\text { hipertrófica. }\end{array}$
\end{tabular}

Caso clínico de mulher de 58 anos hipertensa e 2 apresentação de edema bilateral, ascite, distensão da veia jugular, e ritmo de galope com 3 sons.

Entender os principais aspectos envolvidos na morte súbita destes pacientes, desde a fisiopatologia, a avaliação de risco, a prevenção e as perspectivas futuras.

Neste relatório, o foco estava principalmente em algumas áreas de interesse e desenvolvimento recente na compreensão e gestão de HCM. Restando alguma variabilidade regional no padrão de atendimento disponível.

Definir o papel da ressonância magnética cardíaca na cardiomiopatia hipertrófica.

Discutir a respeito da apresentação clínica da síncope na cardiomiopatia hipertrófica obstrutiva

Explanar os resultados clínicos e evolução ecocardiográfica da miectomia septal estendida na cardiomiopatia hipertrófica obstrutiva.

Apresentação dos resultados do Registo Português de Miocardiopatia Hipertrófica.

Identificar a extensão da necessidade clínica não atendida em pessoas com doenças raras, como $\mathrm{CMH}$ e, assim, determinar resultados relevantes e desfechos de segurança para uso em ensaios.

Descrição do caso de uma paciente de 43 anos que apresentou uma emergência hipertensiva sem história prévia de hipertensão arterial sistêmica.

\section{PRINCIPAIS RESULTADOS}

A morte súbita devido à hipertrofia miocárdica atribuída aos esteróides, conforme descrito anteriormente, é incomum, mas deve ser levada em consideração em pacientes jovens esportistas.

No presente caso, encontramos dimensões aumentadas do VE, função sistólica diminuída, hipertrofia ventricular e fisiologia restritiva, que caracterizam um fenótipo dilatado, restritivo, hipertrófico.

De todos estes marcadores, a história familiar de MS parece ser o de maior relevância, apesar do valor preditivo positivo baixo.

Progresso significativo foi feito em muitos aspectos da compreensão e tratamento da $\mathrm{CMH}$ na última década. Isso se traduz em um melhor cuidado ao lidar com as consequências do processo da doença, como a obstrução da via de saída do ventrículo esquerdo (VSVE) e a previsão e o tratamento de arritmias com risco de vida.

Na difícil tarefa da estratificação de risco para prevenção primária de morte súbita em pacientes com cardiomiopatia hipertrófica, a quantificação da fibrose miocárdica, pela ressonância magnética cardíaca, sugere que indivíduos com risco intermediário pelos fatores de risco clássico possam ser reestratificados.

É preciso considerar a evolução hemodinâmica da CMHO, que pode, por si só, constituir o fator causal para síncope ao esforço, denotando, assim, a evolução desfavorável da classe funcional do quadro em curso.

Quando os pacientes são acompanhados e selecionados para o melhor tratamento disponível por uma equipe multidisciplinar especializada, resultados animadores podem ser obtidos. Isso se traduz em melhora hemodinâmica e clínica dos pacientes em curto prazo e no seguimento, melhorando qualidade de vida para os mesmos.

A mortalidade a longo prazo é baixa; a insuficiência cardíaca é a causa mais comum de morte seguida pela morte cardíaca súbita. No entanto, o fardo da morbidade permanece considerável, enfatizando a necessidade de tratamentos específicos da doença que impactam a história natural da doença.

Em resumo, este estudo identificou as principais necessidades clínicas não atendidas na $\mathrm{CMH}$ e destaca a importância de implementar estratégias aprimoradas de prevenção cardiovascular que aumentem a expectativa de vida das pessoas com $\mathrm{CMH}$.

A CMH é uma doença silenciosa que pode apresentar desde sintomas leves até morte súbita. Devemos sempre considerar esse diagnóstico diante de alterações eletrocardiográficas como HVE e arritmias. 
Comparar o strain longitudinal global (SLG) do ventrículo esquerdo em pacientes portadores de $\mathrm{CMH}$ com e sem HAS associada.

Caso de um homem com quadro de dispneia intensa, ilfiltrado pulmonar, área cardíaca normal e obliteração da ponta do ventrículo esquerdo

Argumentar sobre a terapia médica na insuficiência cardíaca oriunda da cardiomiopatia hipertrofica.

Elucidar as características clínicas, diagnósticas, tratamento e demais aspectos das da cardiomiopatia hipertrófica.

Definir qual a técnica de redução septal mais adequada no caso proposto, cujas características apontam para a necessidade de abordagem invasiva

Compreender as principais manifestações clínicas, diagnóstico e avaliação da cardiomiopatia hipertrofica.

Entender a estratificação de risco para morte cardíaca súbita (MSC). A avaliação do risco de MSC arrítmica é um componente crítico da avaliação clínica de quase todos os pacientes com $\mathrm{CMH}$.

Desmistificar as manifestações clínicas da cardiomiopatia hipestrofica em crianças. A cardiomiopatia hipertrófica (CMH) é uma das formas mais comuns de cardiomiopatia hereditária em adultos e crianças e é caracterizada por hipertrofia do ventrículo esquerdo (HVE), que às vezes envolve o ventrículo direito.

Compreender o gerenciamento de arritimias ventriculares e o risco de morte cardíaca súbita. $\mathrm{O}$ gerenciamento do risco de MSC e arritmias ventriculares em pacientes com CMH está centrado na minimização do risco associado à atividade física e intervenções direcionadas, principalmente implantação de um cardioversor-desfibrilador implantável (CDI) quando indicado.
A deformação do ventrículo esquerdo foi significativamente menor nos hipertensos quando comparada aos normotensos, indicando maior comprometimento da função ventricular naquele grupo. A disfunção diastólica foi melhor caracterizada nos pacientes hipertensos.

Tratar-se de cardiomiopatia de padrão não usual, caracterizada pela superposição de achados da cardiomiopatia hipertrófica e da endomiocardiofibrose. Por outro lado, os achados não eram típicos de nenhuma dessas doenças isoladamente.

Para a maioria dos pacientes com CMH (com ou sem obstrução da VSVE) que permanecem assintomáticos, não usamos rotineiramente terapia medicamentosa profilática antes do início dos sintomas.

Embora muitos pacientes com CMH não apresentem sintomas ou apenas tenham sintomas menores, outros podem apresentar dispneia ao esforço, fadiga, dor no peito, pré-síncope e síncope, durante ou logo após o esforço, e palpitações

O Dr. Hartzell Shaff propõe a miectomia septal cirúrgica como o procedimento mais adequado, e o Dr. Pedro Trujillo propõe a ablação septal percutânea como a solução de escolha

Apesar de um grande número de pacientes com CMH permanerem assintomáticos, não é incomum que desenvolvam um ou mais dos seguintes sintomas: dispneia aos esforços, ortopneia, dispneia paroxística noturna, dor torácica, palpitações, pré-síncope / síncope, tontura postural, fadiga ou edema.

Pacientes com cardiomiopatia hipertrofica têm um risco aumentado de morte por várias causas, incluindo MSC e IC. Os principais fatores de risco e modificadores de risco estabelecidos para MSC incluem: Parada cardíaca prévia ou arritmias ventriculares sustentadas, história familiar de primeiro grau ou parente próximo $<50$ anos de idade.

Os achados clínicos associados à CMH podem se desenvolver em bebês e crianças pré-púberes, mas são mais comumente vistos em adolescentes e adultos jovens após o surto de crescimento e outras alterações associadas à puberdade.

Pacientes que sobrevivem a um episódio de taquicardia ventricular sustentada (TV), recomendamos o implante de um CDI para a prevenção secundária de MSC. O CDI é a melhor terapia disponível para pacientes com $\mathrm{CMH}$ que sobreviveram a parada cardíaca por TV ou que apresentam alto risco de arritmias ventriculares. 
Interpretar as variantes morfológicas e a fisiopatologia da obstrução do trato de saída do ventrículo esquerdo. Pode haver uma variabilidade significativa no grau de obstrução da VSVE na CMH.

A população em estudo foi constituída por 90 pacientes consecutivos com CMH, com idade média de $62 \pm 12$ anos, $85(94 \%) \geq 40$ anos e $56(62 \%)$ do sexo feminino. No período médio de seguimento de $6 \pm 3$ anos, 15 (17\%) implantaram CDI para prevenção primária de MS. Dois (2\%) pacientes apresentaram choque apropriado, 6 (7\%) MS e $6(7 \%)$ morte por outras causas.

O objetivo desta revisão narrativa é enfocar as cardiomiopatias mais importantes, sua epidemiologia, seus aspectos genéticos, diagnóstico e manejo.

Avaliar o perfil de jovens adultos (16-25 anos) com HCM incluídos no registro prospectivo de $\mathrm{HCM}$ francês.

Descrever as características e os resultados da $\mathrm{CMH}$ primária com início na infância em uma grande coorte multicêntrica. Este estudo se concentra em pacientes diagnosticados entre 1 e 18 anos de idade, descrevendo resultados cardíacos adversos e preditores de risco.

Nosso estudo ressalta a importante contribuição da epigenética e do meio ambiente na progressão da doença em pacientes com $\mathrm{CMH}$ diagnosticados geneticamente.
A obstrução da VSVE ( $\geq 30 \mathrm{mmHg}$ ) em repouso está presente em até 30 por cento dos pacientes com cardiomiopatia hipertrófica $(\mathrm{CMH})$, enquanto outros 30 por cento não têm evidência de obstrução da VSVE em repouso, mas desenvolvem obstrução da via de saída com provocação.

Foi identificada entre os critérios de prevenção primária de MS estabelecidos pelas diretrizes ACCF/AHA 2011 e ESC 2014. O escore ESC HCM-RiskSCD reduziu as indicações para implante de CDI na população estudada, notadamente naqueles situados em Classe IIa na sistematização norte-americana, mas deixou desprotegida a totalidade de pacientes que sofreram MS ou choque apropriado.

Foi relatado que a HCM é a causa mais importante de morte súbita no campo de atletismo nos Estados Unidos.

Em adultos jovens com $\mathrm{CMH}$, os MACE são comuns em curto prazo, especialmente em $\mathrm{CMH}$ obstrutiva e mulheres, principalmente de origem arrítmica. $\mathrm{O}$ implante profilático de CDI é frequente e não segue estritamente as diretrizes, enquanto o uso das diretrizes europeias / americanas é útil, mas imperfeito na identificação do risco de MSC.

A $\mathrm{CMH}$ de início na infância têm diferenças importantes nas características clínicas, genótipo e desfechos cardiovasculares. Eles são mais propensos a ter doença sarcomérica, têm um risco maior de arritmias ventriculares e uma frequência maior de IC avançada do que os pacientes diagnosticados na idade adulta.

Os dados inferem um papel crítico para os fatores ambientais na determinação da progressão morfológica cardíaca em pacientes com CMH. Presumivelmente, as consequências de diferentes fatores ambientais são mediadas por mudanças no epigenoma.

Fonte: Elaboração própria.

\section{Discussão}

Vale ressaltar que a CMH, como exemplificado por Martin S. Maron (2020), trata-se de uma doença do músculo cardíaco de caráter eminentemente genético, sendo causada mais frequentemente (60 a 70 por cento) por mutações em um dos vários genes do sarcômero que codificam componentes do aparelho contrátil do coração.

Após análise, observou-se que a doença em questão é caracterizada por hipertrofia ventricular esquerda (HVE) de morfologias diversas e com um leque de manifestações clínicas e anormalidades hemodinâmicas a depender do local e da extensão da hipertrofia cardíaca, podendo, inclusive variar dentro de uma mesma família. Por conseguinte, os pacientes com CMH podem desenvolver uma ou mais das seguintes anormalidades: obstrução do fluxo de saída do ventrículo esquerdo, disfunção diastólica, isquemia do miocárdio e insuficiência mitral (Maron, 2020).

Nessa perspectiva, os sintomas associados incluem a apresentação assintomática, morte súbita cardíaca (MSC), arritmias ventriculares, obstrução, insuficiência cardíaca com fração de ejeção preservada ou reduzida, fibrilação atrial e acidente vascular encefálico (AVE). Mesmo que uma gama de pacientes com CMH não apresentem sintomas ou apenas 
tenham sintomas menores, outros podem apresentar dispneia (devido à disfunção diastólica, particularmente durante o exercício), fadiga, dor no peito (devido à redução da reserva coronariana, doença microvascular e aumento da demanda de oxigênio), pré-síncope e síncope, durante ou logo após o esforço e palpitações (Bazan et al., 2020).

Um ponto de extrema importância na CMH e com uma apresentação catastrófica é a MSC, resultante principalmente de arritmias ventriculares, obstrução dinâmica da VSVE, isquemia miocárdica (a despeito da ausência de doença coronariana aterosclerótica), desarranjo dos miócitos, fibrose miocárdica e, em menor proporção, colapso hemodinâmico agudo caracterizado por insuficiência cardíaca e hipotensão grave. Vale sublinhar que o grau de obstrução não se correlaciona estritamente com a gravidade dos sintomas ou risco de MSC (Fernandes, 2018).

Importante trazer a luz que a MSC comporta-se como a primeira manifestação clínica da CMH em diversos casos. Todavia, nas demais situações os principais fatores de risco para MSC são: história pregressa de parada cardíaca, fibrilação ventricular ou taquicardia ventricular sustentada. Tais fatores podem levar a uma mortalidade aproximada de $10 \%$ ao ano no referido grupo, como mostrado pela Sociedade Brasileira de Cardiologia (SBP, 2018). Porém, é sabido que a MSC que mais repercute atenção advém de casos assintomáticos e sem história prévia de alterações cardíacas, sendo em sua grande maioria classificados em grau I e II pela New York Heart Association (NYHA) como exemplificado na abela 6, daí o seu grau de relevância. Nesse cenário, estudos recentes apontam que a CMH é a principal causa de MSC em adultos jovens com menos de 35 anos, sejam eles, atletas profissionais ou amadores.

Tabela 6: Classificação New York Heart Association.

\begin{tabular}{cl}
\hline CLASSE & CLASSIFICAÇÃo FUNCIONAL NYHA \\
\hline I $\quad \begin{array}{l}\text { Pacientes com doença cardíaca, mas sem limitações resultantes da atividade física. A atividade física comum não causa fadiga } \\
\text { indevida, palpitações, dispneia ou dor anginosa. }\end{array}$ \\
II $\quad \begin{array}{l}\text { Pacientes com doença cardíaca resultando em ligeira limitação da atividade física. Eles se sentem confortáveis em repouso. A } \\
\text { atividade física comum resulta em fadiga, palpitações, dispneia ou dor anginosa. }\end{array}$ \\
III $\quad \begin{array}{l}\text { Pacientes com doença cardíaca resultando em limitação acentuada da atividade física. Eles sentem confortáveis em } \\
\text { repouso. A atividade física incomum causa fadiga, palpitações, dispneia ou dor anginosa. }\end{array}$ \\
Pacientes com doenças cardíacas que resultam na incapacidade de realizar qualquer atividade física sem desconforto. Os \\
sintomas de insuficiência cardíaca ou da síndrome anginosa podem estar presentes mesmo em repouso. Se qualquer atividade \\
física for realizada, o desconforto aumenta.
\end{tabular}

Fonte: O Comitê de Critérios da New York Heart Association. Nomenclatura e critérios para o diagnóstico de doenças do coração e grandes vasos, (9.ed,) Little, Brown \& Co, Boston 1994. p.253.

Sob um ponto de vista semiológico, a $\mathrm{CMH}$ pode apresentar tanto achados normais, quanto um quadro polissintomático, por exemplo, presença de quarta bulha cardíaca (B4), sopro sistólico de regurgitação na borda esternal esquerda inferior, desdobramento paradoxal da segunda bulha cardíaca (B2), ictus cordis aumentado e frêmito sistólico. De forma complementar, pacientes mais graves que possuem fração de ejeção do ventrículo esquerdo reduzida podem apresentar o sopro sistólico de ejeção, tornando-se mais audível ao levantar da posição de cócoras ou através do emprego da manobra de Valsalva e, frequentemente, irradiado para borda superior direita (Bazan, 2020). 
Research, Society and Development, v. 10, n. 13, e336101321498, 2021

(CC BY 4.0) | ISSN 2525-3409 | DOI: http://dx.doi.org/10.33448/rsd-v10i13.21498

\section{Conclusão}

O presente estudo visa enriquecer a literatura e enfatizar o déficit de produções científicas a respeito das manifestações clínicas da CMH. Atentos a todos os pontos aqui elencados, subtende-se que a CMH é uma patologia de alta relevância para a medicina, haja vista sua característica de manifestação silenciosa, apresentando morfologias diversas e uma série de anormalidades hemodinâmicas, variando até mesmo em um núcleo familiar, merecendo assim, devida atenção. Deste modo, a maior dificuldade encontra-se em como determinar os pacientes que apresentam maior risco de desenvolver obstrução dinâmica da VSVE, arritmias ventriculares graves, IC e MSC.

Portanto, a triagem eficaz torna-se importante e essencial a fim de detectá-la precocemente. Isso possibilita uma intervenção clínica antecipada, no intuito de garantir melhor prognóstico e qualidade de vida aos pacientes, de modo a afastar principalmente os desfechos mais trágicos da doença em questão, bem como complicações incapacitantes.

Por fim, fica como sugestão para pesquisas futuras abordagens voltadas para prevalência quantitativa das manifestações clínicas e sua relação com as alterações genéticas da patologia em questão. De modo, que possamos identificar as manifestações mais comuns conforme a carga genotípica, podendo então, melhorar cada vez mais a o diagnóstico precoce desses pacientes, possibilitando melhoria na qualidade de vida dos mesmos, bem como evitar desfechos trágicos, como MSC principalmente em jovens.

\section{Referências}

Arias, A. M., Arenaza, P. R., Marenchino, R. G., Garagoli, F., Rivello, H. G. \& Belziti, C. (2018). Cardiomiopatia Hipertrófica, Todos os Fenótipos em um. International Journal of Cardiovascular Sciences. 31(3)312-315. https://pesquisa.bvsalud.org/portal/resource/pt/biblio-909037

Baron, Émilie., Karam, N., Donal, E., Puscas, T., Mirabel, M., Bacher, A., Wahbi, K., Mazzella, JM., Jeunemaitre, X., Reant, P., Hagège, A. (2021). Gestion et résultats de la cardiomyopathie hypertrophique chez les jeunes adultes. Archives of Cardiovascular Diseases, 114(6-7): 465-473. https://sfcardio.fr/sites/default/files/2021-06/2021_06-article_ACVD.pdf

Bazan, S. G. Z., Oliveira, G. O., Silveira, C. F. S. M. P. , Reis, F. M., Malagutte, K. N. D. S., Tinasi, L. S. N., Bazan, R., Hueb, J. C., \& Okoshi, K. (2020). Cardiomiopatia Hipertrófica - Revisão. Arq. Bras. Cardiol., 115(5), 927-935. https://doi.org/10.36660/abc.20190802

Bittencourt, M. I., Cader, S. A., Araúlo, D. V., Salles, A. 1. F., Albuquerque, F. N., Spineti, P. P. M., Albuquerque, D. C. \& Rocha, R. M. (2016). Morte Súbita na Cardiomiopatia Hipertrófica. International Journal of Cardiovascular Sciences, 29(6):504-511. https://pesquisa.bvsalud.org/portal/resource/pt/biblio832441

Braunwald, E. (2013). Braunwald: Tratado de Doenças Cardiovasculares: Cardiomiopatia Hipertrófica. In: Cardiomiopatia Hipertrófica. [S. 1.: s. n.], 2013. cap. 69 , p. 1620.

Braunwald, E. (2013). Braunwald: Tratado de Doenças Cardiovasculares: Causas Hereditárias da Doença Cardiovascular. In: Causas Genéticas da Cardiomiopatia. [S. 1.: s. n.], 2013. Cap. 8, p. 72

Braunwald, E. (2013). Braunwald: Tratado de Doenças Cardiovasculares: Causas Hereditárias da Doença Cardiovascular. In: Mutações de genes da proteína do sarcômero. [S. 1.: s. n.], 2013. cap. 8, p. 72.

Brown \& Co (1994). O Comitê de Critérios da New York Heart Association. Nomenclatura e critérios para o diagnóstico de doenças do coração e grandes vasos, $9^{a}$ ed, Little, Boston 1994. p.253

Cardim, N., Brito, D., Lopes, L.R., Freitas, A., Araújo, C., Belo, A., Gonçalves, L., Mimoso, J., Olivotto, I., Elliott, P., Madeira, H. (2018). The Portuguese Registry of Hypertrophic Cardiomyopathy: Overall results. Revista Portuguesa de Cardiologia, 37(1): 1-10. https://www.sciencedirect.com/science/article/pii/S0870255117305425?via\%3Dihub

Cardiomiopatia hipertrófica: abordagem clínica e opções de tratamento. (2020). Uruguai Journal of Cardiology , 35 (1), $290-348$. Epub 01 de abril de 2020. https://dx.doi.org/10.29277/cardio.35.1.15

Ciarambino, T., Menna, G., Sansone, G., Giordano, M. (2021). "Cardiomyopathies: An Overview". International Journal of Molecular Sciences, 22(14): 7722. https://www.mdpi.com/1422-0067/22/14/7722/htm

Conde, M. D., Miranda, D. B., Villegas, F. G. M. \& Pliego, R. R. H. (2017). Parada cardíaca súbita atribuída a cardiomiopatia hipertrófica devido ao uso de esteróides anabolizantes. Relato de caso. Critical Medicine (Mexican College of Critical Medicine), 31 (2), 101-105. http://www.scielo.org.mx/scielo.php?script=sci_arttext\&pid=S2448-89092017000200101\&lng=es\&tlng=es

Cooper, R.M., Raphael,C.E., Liebregts, M., Anavekar, N.S., Veselka, J. (2017). New Developments in Hypertrophic Cardiomyopathy. Canadian Journaul of Cardiology, 33(10): 1254-1265. https://www.onlinecjc.ca/article/S0828-282X(17)30371-9/fulltext 
Research, Society and Development, v. 10, n. 13, e336101321498, 2021

(CC BY 4.0) | ISSN 2525-3409 | DOI: http://dx.doi.org/10.33448/rsd-v10i13.21498

Duarte, K. M., Duarte, V. S., Bezerra, A. L. D., Feitosa, A. A. N., Assis, E. V. \& Sousa, M. N. A. (2014). Dificuldades encontradas para a implantação da educação popular na realização da promoção de saúde. Revista Interdisciplinar em Saúde, Cajazeiras, v. 1, n. 1, p.33-51.

Fernandes, F. V., Bello, J. H. S. M., Shiozaki, A. A. \& Roberto, C. (2018). Papel Atual da Ressonância Magnética Cardíaca na Cardiomiopatia Hipertrófica. Arq Bras Cardiol: Imagem cardiovasc, 31(4):277-283. https://pesquisa.bvsalud.org/portal/resource/pt/biblio-964042

Gil, T. C. P, Castier, M. C., Gondar, A. F. P., Sales, A. F., Santos, M. O., Lima, F. C. S. \& Rocha, R. M. (2019). Análise de Strain da Função Ventricular Esquerda na Associação de Cardiomiopatia Hipertrófica e Hipertensão Arterial Sistêmica. Arquivos Brasileiros de Cardiologia, v. 113, n. 4. https://doi.org/10.5935/abc.20190176

Jefferies, J. L., Ryan, T. D. \& Maron, M. S. (2020). Hypertrophic cardiomyopathy in children: Clinical manifestations and diagnosis. In Triedman J K, ed. UpToDate. Waltham, Mass.:UpToDate. https://www.uptodate.com/contents/hypertrophic-cardiomyopathy-in-children-clinical-manifestations-anddiagnosis?search=manifesta\%C3\%A7\%C3\%B5es\%20clinicas\%20na\%20 ardiomiopatia\%20hipertrofica\&source=search_result\&selectedTitle=2 150\&usage _type $=$ default\&display_rank $=2$

Kumar, V. (2020). Robbins \& Cotran Patologia: Bases Patológicas das Doenças: O Coração. In: CARDIOMIOPATIA Hipertrófica. 9ª ed. [S. 1.: s. n.], 2020. cap. 12, p. 1026.

Kumar, V. (2020). Robbins \& Cotran: Patologia: Bases Patológicas das Doenças: O Coração. In: CARDIOMIOPATIA Hipertrófica. $9^{\text {a }}$. ed. [S. 1.: s. n.], 2020. cap. 12, p. 1025.

Licarião, E. G. D. (2018). Síncope na cardiomiopatia hipertrófica obstrutiva. JBAC, 31(3):121-5. https://pesquisa.bvsalud.org/portal/resource/pt/biblio-967814

Loss, F. S., Ueda, L. S. I. \& Luiz, A. A. (2019). Cardiomiopatia hipertrófica. Relato de caso. Uruguai Journal of Cardiology , 34 (3), 184-196. Epub 01 de dezembro de 2019. https://dx.doi.org/10.29277/cardio.34.3.16

Ludke, M. \& Andre, M. E. D. A. (2013). Pesquisas em educação: uma abordagem qualitativa. São Paulo: E.P.U

Maron, M. S. (2019). Hypertrophic cardiomyopathy: Medical therapy for heart failure. In McKenna W J, ed. UpToDate. Waltham, Mass.:UpToDate, 2019. https://www.uptodate.com/contents/hypertrophic-cardiomyopathy-medical-therapy-for-heart-

failure?search=Hypertrophic\%20 cardiomyopathy:\%20Medical\%20therapy\%20for\%20heart\%20failure\&source=search_result\&selectedTitle=1 150\&usage_t ype=default\&display_rank $=1$

Maron, M. S. (2020). Hypertrophic cardiomyopathy: Clinical manifestations, diagnosis, and evaluation. In McKenna W J, ed. UpToDate. Waltham, Mass.:UpToDate. https://www.uptodate.com/contents/hypertrophic-cardiomyopathy-clinical-manifestations-diagnosis-and-evaluation?source=history_widget

Maron, M. S. (2020). Hypertrophic cardiomyopathy: Management of ventricular arrhythmias and sudden cardiac death risk. In Lévy S, McKenna W J, ed. UpToDate. Waltham, Mass.:UpToDate. https://www.uptodate.com/contents/hypertrophic-cardiomyopathy-management-of-ventricular-arrhythmias-andsudden-cardiac-death-risk?search=manifesta\%C3\%A7\%C3\%B5es\%20clinicas\%20na\%20cardiomiopatia\%20hipertrofica\&topicRef=4951\&source=see_link

Maron, M. S. (2020). Hypertrophic cardiomyopathy: Morphologic variants and the pathophysiology of left ventricular outflow tract obstruction. In McKenna W J, ed. UpToDate. Waltham, Mass.:UpToDate. https://www.uptodate.com/contents/hypertrophic-cardiomyopathy-morphologic-variants-and-thepathophysiology-of-left-ventricular-outflow-tract-

obstruction?search=manifesta\%C3\%A7\%C3\%B5es\%20clinicas\%20na\%20cardiomiopatia\%20hipertrofica\&topicRef=119625\&source=see_link

Maron, M. S. (2020). Hypertrophic cardiomyopathy: Risk stratification for sudden cardiac death. In Lévy S, McKenna W J, ed. UpToDate. Waltham, Mass.:UpToDate.

death?topicRef=4948\&source=see_link https://www.uptodate.com/contents/hypertrophic-cardiomyopathy-risk-stratification-for-sudden-cardiac-

Marston, N. A., Han, L., Olivotto, I., Day, S. M., Ashley, E. A., Michels, M., Pereira, A. C., Ingles, J., Semsarian, C., Jacoby, D., Colan, S. D., Rossano, J. W., Wittekind, S.G., Ware, J. S., Saberi, S., Helms, A. S., Ho, C.Y. (2021). Clinical characteristics and outcomes in childhood-onset hypertrophic cardiomyopathy. European Heart Journal, 42(20): 1988-1996. https://academic.oup.com/eurheartj/article/42/20/1988/6189026

Mattos, B. P. (2001). Bases Genéticas da Cardiomiopatia Hipertrófica. Arq Bras Cardiol, Porto Alegre, v. 78, ed. 3, p. 323, 26 mar.

Mattos, B. P., Scolari, F. L. \& Garbin, H. I. (2020). Discordância entre Diretrizes Internacionais sobre Critérios de Prevenção Primária de Morte Súbita na Cardiomiopatia Hipertrófica. Arq Bras Cardiol., 115(2):197-204. https://pesquisa.bvsalud.org/portal/resource/pt/biblio-1131297

Pujades-Rodriguez, M., Guttmann, O.P., Gonzalez-Izquierdo, A., Duyx, B., O’Mahony, C., Elliott, P., Hemingway, H. (2018). Identifying unmet clinical need in hypertrophic cardiomyopathy using national electronic health records. PloS ONE, 13(1): e0191214. https://journals.plos.org/plosone/article?id=10.1371/journal.pone.0191214

Repetti, G.G., Kim, Y., Pereira, A.C., Ingles, J., Russell, M.W., Lakdawala, N.K., Ho, C.Y., Day, S., Semsarian, C., McDonough, B., DePalma, S.R., Quiat, D., Green, E.M., Seidman, C.E., Seidman, JG. (2021). Discordant clinical features of identical hypertrophic cardiomyopathy twins. PNAS, 118(10): e2021717118. https://www.pnas.org/content/118/10/e2021717118

Sarli, I. V. \& Benvenuti, L. A. (2019). Case 3/2019 - Jovem macho com Dispneia Intensa, Infiltrado Pulmonar, Área Cardíaca Normal e Obliteração da Porção Apical do Ventrículo Esquerdo. Arquivos Brasileiros de Cardiologia [online]. 2019, v. 112, n. 6. https://doi.org/10.5935/abc.20190105

Vrancic, J. M., Costabel, J. P., Espinola, J. C., Piccinini, F., Camporrotondo, M., Pedernera, G. O., Avegliano, G., Diez, M., Dorsa, A. \& Navia, D. (2018). Miectomía septal ampliada en la miocardiopatía hipertrófica obstructiva, resultados clínicos y evolución ecocardiográfica a mediano plazo. Rev ARgent CARdiol, 86:96-102. https://pesquisa.bvsalud.org/portal/resource/pt/biblio-1003184 